\title{
Challenges in Developing Novel Treatments for Childhood Disorders: Lessons from Research on Anxiety
}

\author{
Daniel S Pine ${ }^{*, 1}$, Sarah M Helfinstein ${ }^{2}$, Yair Bar-Haim ${ }^{1,3}$, Eric Nelson ${ }^{1}$ and Nathan A Fox ${ }^{1,2}$ \\ ${ }^{1}$ Mood and Anxiety Disorders Program, Intramural Research Program, The National Institute of Mental Health, Bethesda, MD, \\ USA; ${ }^{2}$ Department of Human Development, University of Maryland, College Park, MD, USA; ${ }^{3}$ Tel Aviv University, Tel Aviv, Israel
}

\begin{abstract}
Alterations in brain development may contribute to chronic mental disorders. Novel treatments targeted toward the earlychildhood manifestations of such chronic disorders may provide unique therapeutic opportunities. However, attempts to develop and deliver novel treatments face many challenges. Work on pediatric anxiety disorders illustrates both the inherent challenges as well as the unusual opportunities for therapeutic advances. The present review summarizes three aspects of translational research on pediatric anxiety disorders as the work informs efforts to develop novel interventions. First, the review summarizes data on developmental conceptualizations of anxiety from both basic neuroscience and clinical perspectives. This summary is integrated with a discussion of the two best-established treatments, cognitive behavioral therapy and selective serotonin reuptake inhibitors. Second, the review summarizes work on attention bias to threat, considering implications for both novel treatments and translational research on neural circuitry functional development. This illustrates the manner in which clinical findings inform basic systems neuroscience research. Finally, the review summarizes work in basic science on fear learning, as studied in fear conditioning, consolidation, and extinction paradigms. This summary ends by describing potential novel treatments, illustrating the manner in which basic neuroscience informs therapeutics. Neuropsychopharmacology Reviews (2009) 34, 213-228; doi: I 0.1038/npp.2008.I I3; published online 27 August 2008
\end{abstract}

Keywords: anxiety; children; adolescents; amygdala; attention; conditioning

\section{INTRODUCTION}

Risk for chronic mental disorders relates to individual differences in brain development and early-life behavior. As many mental illnesses start during childhood, treatment of childhood problems may have a better hope of reducing long-term morbidity than treatments targeted toward adult problems. Unfortunately, the current dearth of treatments for pediatric mental disorders reflects complex challenges. Recent research on pediatric anxiety disorders highlights many of these complexities. Moreover, in anxiety, attempts to find novel therapies benefit from knowledge of pathophysiology emerging in light of strong cross-species parallels. Across mammals, 'fear' represents a brain state engaged by acute, immediately present 'threats', stimuli that the organism will extend effort to avoid; 'anxiety' represents

${ }^{*}$ Correspondence: Dr DS Pine, Mood and Anxiety Disorders Program, National Institute of Mental Health, 15K North Drive, MSC-2670, Bethesda, MD 20892-2670, USA, Tel: +1 301594 1318, Fax: +1 301 402 2010, E-mail: Daniel.pine@nih.gov

Received 10 March 2008; revised 25 April 2008; accepted 28 April 2008 a brain state engaged when encountering sustained cues that more ambiguously predict threat. Across species, individual differences in fear and anxiety reflect individual differences in shared neural architecture. Research in other areas of medicine suggests that efforts to generate novel treatments benefit from a mutually reinforcing dialogue among basic and clinical researchers that is organized around observations of cross-species parallels in relevant biology.

The present review unfolds in three stages. The review begins by summarizing developmental conceptualizations of anxiety in both clinical and basic arenas. Opportunities and complications are then discussed associated with the two beststudied therapies, cognitive behavioral therapy (CBT), and selective serotonin reuptake inhibitors (SSRIs). Second, the review summarizes clinical findings that carry basic implications. Specifically, this summary delineates the manner in which attention orienting toward threat relates to both clinical aspects of anxiety as well as the associated neural circuitry. Thus, this portion of the review illustrates the relevance of clinical findings for research in basic neuroscience. Finally, the review describes findings in animal models, demonstrating how neuroscience informs therapeutics. 


\section{DEVELOPMENTAL CONCEPTUALIZATION OF ANXIETY}

\section{Neuroscience and Classification}

Various schemes have been used to classify individual differences in pediatric anxiety. For research on therapeutics, most work uses the Diagnostic and Statistical Manual to identify youths with generalized anxiety disorder, separation anxiety disorder, or social phobia. As a result, the present review focuses on only these three conditions but no other anxiety disorders. Owing to comorbidity, most therapeutic studies actually target individuals with any one of these conditions (Bridge et al, 2007). As reviewed elsewhere (Hyman, 2007; Pine, 2007), these comorbidity data, coupled with preliminary data in neuroscience, suggest the need to revise classification. Emerging findings suggest that distinct pediatric anxiety disorders reflect stable individual differences in at least three sets of dissociable information-processing functions (Pine, 2007). For example, one set of information-processing functions relates to individual differences in the identification and appraisal of specific threats, such as angry peers, which in turn relate to disorder-specific clinical features, such as social phobia and social anxiety more generally. Other information-processing functions relate, more broadly, to individual differences in how a wider range of threats, once identified, are cognitively processed, as occurs in virtually all anxiety disorders (Bar-Haim et al, 2007; Lissek et al, 2005).

The current summary can be viewed as a companion paper to an earlier review (Pine, 2007). This earlier review describes how specific information-processing functions relate to both general and specific risks for and expressions of particular pediatric anxiety disorders. The present review, in contrast, focuses on therapeutics. Available data suggest that current treatments target perturbations present across generalized anxiety disorder, separation anxiety disorder, social phobia, and risk factors for these conditions, considered together as a group (Rapee et al, 2005). As a result, the present review focuses on informationprocessing functions implicated in a range of anxiety states but does not discuss particular disorder-specific features. Basing future nosology on clinically relevant informationprocessing measures will facilitate classification grounded in neuroscience. This will be possible, given the precise manner in which information-processing functions have been linked to neural system function (Pine, 2007). More importantly, however, a focus on neuroscience can generate novel therapeutic insights.

This review summarizes data on attention and learning. These information-processing functions have been linked to both individual differences in threat-response behavior and brain function. However, their roles may be developmentally dissociable. For example, because attention may relate to individual differences, manifest very early, understanding in this area may inform treatments targeted to the earliestoccurring subclinical aspects of anxiety disorders. In contrast, for other, learning-related processes, such as extinction of acquired fears, relations to individual differences reflect variations in the ability to manage or control already well-established anxiety states, manifest in relatively mature individuals. Thus, understanding in this area may carry implications for treatment of firmly established anxiety disorders.

\section{Longitudinal Data}

Data show that most adults with anxiety disorders, as well as mood disorders, manifest anxiety early in life (Pine, 2007). Research on temperament suggests that such risk for adult mood and anxiety disorders can be detected very early, even before school-age (Kagan, 1994), and can predict risk through adulthood (Beesdo et al, 2007; Perez-Edgar and Fox, 2005). Thus, if early anxiety is effectively treated, this could impact both children and adults. Opportunities for developing novel treatments appear particularly ripe in pediatric anxiety, given data in various mammalian species showing that early-life events influence individual differences in the threat responses of adults.

Although longitudinal data suggest the potential importance of therapeutics, they also point to potential complications. Early emerging individual differences in anxious behavior represent only one contributor to later-life anxiety disorders; pediatric anxiety is not invariably a chronic, recurrent condition, as most children with anxiety will not manifest clinical conditions as adolescents or adults. Moreover, the relations between early-life variations in anxious behavior and risk for later-life clinically significant disorders appear continuous, which complicates efforts to categorically set a boundary that identifies children as unequivocally in need or not in need of treatment. If a boundary is set too high, at-risk children are missed; if it is set too low, children are treated unnecessarily, exposing them to risks.

Finally, therapeutic decisions rely heavily on clinical judgments about impairment; a reasonable approach given that delivery of potentially risky treatment seems justifiable in a child whose anxiety precludes normal functioning. However, because impairment is influenced by factors external to the child, current practice dictates that clinicians view differently two children with identical biological predispositions. This practice will change in an age when treatment decisions rely on an integration of data from initial judgments, based on clinical evaluation, with data on pathophysiology, as occurs in other areas of medicine. Given current knowledge, however, research in neuroscience appears most relevant for testing hypotheses about novel treatment, forcing current treatment decisions to remain grounded only in clinical judgment.

\section{Available Treatments}

Data in neuroscience generate insights on the two beststudied treatments: CBT and SSRIs. Neuroscience generates insights on both beneficial and adverse outcomes. 
CBT. The utility of CBT in anxiety provides insights on pathophysiology and treatment (Bouton, 2002; Quirk and Mueller, 2008). CBT works by first teaching anxious children cognitive strategies to employ when encountering threats and then guiding these children through exposures to increasingly extreme threats. Two aspects of CBT inform current thinking.

First, teaching anxious children strategies for controlling their attention represents one important technique for reducing anxiety. This technique is thought to address biased attention allocation toward threats (see below). Neuroscience data demonstrate that both implicit and explicit factors influence attention. Attention-retraining in CBT targets explicit control over attention by directly teaching children techniques for reallocating their attention when anxious. Novel treatment approaches might emerge by augmenting such explicit training with implicit attention retraining, using repeated exposure to events that induce plasticity even when no directed instruction is provided to children about techniques for 'retraining' attention.

Second, CBT models the process of fear extinction. Basic science work suggests that extinction involves new learning as opposed to forgetting, conceptualized as passive fading of threat-related associations. Hence, extinction involves acquiring new memories where stimuli-safety associations become more salient (Bouton, 2002).

Longitudinal data resonate with data on CBT and extinction. Most pediatric disorders remit, suggesting that extinction-like processes represent an expectable phase of development. Viewed from this perspective, pediatric anxiety disorders can be separated into those that remit and those that persist, due to failures to extinguish, with the latter group accounting for most adult mood and anxiety disorders. Parenthetically, this view raises questions on the boundaries of normative behavior. Although extreme anxiety is distressing and often impairing, many children exhibit this problem, and it is usually transient. Is this normative? The less common form of anxiety that fails to extinguish throughout development is more clearly viewed as abnormal.

Findings in rodents and nonhuman primates suggest the potential importance of delivering CBT to anxious children. This work shows that manipulations exposing young organisms to stress produce long-term effects on threatresponse behavior (Gross and Hen, 2004; Meaney, 2001). Because effects are more sustained in juvenile than mature organisms, these data bring to the forefront conundrums about balancing risk and benefit in therapeutics. Nevertheless, clinical relevance remains relatively indirect. Most work in animals examines effects during developmental periods analogous to early childhood, before most children with anxiety disorders present for treatment. Indirectly, such work in animals still remains relevant for views of pediatric anxiety disorder therapy. This is because stressresponse systems in humans exhibit signs of marked plasticity well into adolescence, a period where data on stress-response development in animal systems remain limited (Gunnar and Quevedo, 2007); in animal models, the precise time window during which age-related plasticity ends has not been delimited. Thus, a one-to-one mapping is not possible, linking stress-related influences on rodent, nonhuman primate, and human development. Broadly viewed, however, data on the sustained effects of early-life stress raise the possibility that early treatment influences adult outcome. These data raise questions on risk-benefit ratios. Although attention has been focused on the potential risks of early SSRIs, attention might also consider the risks of CBT, given that a key component of CBT involves exposing children to threats, which generates considerable stress in the child.

Application of CBT to children raises question on possible adverse effects, particularly in light of efforts to deliver CBT to increasingly younger anxious children (Rapee et al, 2005). CBT is explicitly designed to be at least mildly stressful, as the exposure components require the child to experience some degree of distress. Available longterm data in older children find no adverse effects (Barrett et al, 2001). Nevertheless, data on the enduring effects of stress lay bare one major hurdle complicating research on therapeutics with children. Children are more vulnerable than adults, not only because they depend on adults to act on their behalf, but also because manipulations during childhood exert more enduring effects than manipulations in adulthood on brain systems engaged by emotionally evocative experiences. As demonstrated in rodents, these enduring effects occur following both psychological and chemical manipulations. This emphasizes the sensitive nature of treatment decisions in children.

Thus, on the one hand, the vulnerable state of the child necessitates a cautious approach to therapeutics. On the other hand, the plastic state of the immature organism provides a unique opportunity to alter long-term trajectories of pathological fear-related behaviors. A balancing of these unique vulnerabilities and opportunities, which appear particularly well defined in pediatric anxiety, complicates virtually all pediatric research on novel therapeutics.

SSRIs. SSRIs represent the second efficacious treatment in pediatric anxiety. As with CBT, data for SSRIs raise questions both on factors associated with pathophysiology and treatment. At one level, the effect of SSRIs on pediatric anxiety appears particularly robust (Bridge et al, 2007). Strong efficacy data and data implicating serotonin in anxiety more generally support recommendations of treating pediatric anxiety with SSRIs. Available data in neuroscience also raise broad questions on the role of serotonin in development.

As with data on early stress effects, data on serotonin carry relatively indirect clinical implications. This reflects the fact that effects in mice appear most robust in young, preweanlings (Ansorge et al, 2008), coupled with the fact that the precise developmental time window during which serotonin can exert uniquely robust effects on anxiety remains imprecisely specified. Nevertheless, as with 
research on early-life stress, few studies examine serotonin effects in adolescent rodents, and some suggest that stresssensitive systems in the human exhibit plasticity that extends further into development than in the rodent (Gunnar and Quevedo, 2007). Regardless, these data, like data on stress exposure, also raise essential questions on early opportunities and risks that frame clinical risk-benefit balancing. Specifically, genetic and pharmacological manipulations in animal models show that the relations between serotonin and anxiety evolve in a developmental context. For example, in mice, serotonin manipulations increase anxious behavior only when occurring in a specific time window. On the other hand, treating immature rodents with SSRIs attenuates negative effects of stress (Ishiwata et al, 2005). Moreover, even in mature organisms, SSRIs might restore plasticity typical of early development (Maya Vetencourt et al, 2008). Thus, serotonin interacts with stress-related and developmental processes in complex, incompletely understood ways that influence anxiety.

Given cross-species parallels, mechanistic understanding of serotonin-anxiety relations in rodents may carry implications for humans. Serotonin manipulations in the developing rodent produce relatively heterogeneous behavioral effects (Gross and Hen, 2004). Thus, serotonin may exert heterogeneous effects in humans, influencing one set of particular behaviors or individuals more than others. Moreover, individual differences in pediatric anxiety evolve as the brain matures. Serotonin influences also may vary as a function of these developmental processes.

Much in the manner that data on stress exposure frame risk-benefit analysis for CBT, data on serotonin manipulations inform risk-benefit analysis in SSRIs. However, as with CBT, the lack of explicit clinical implications from basic work forces current treatment decisions to remain grounded in clinical data. Although long-term SSRI effects in immature rodents emphasize the need for caution, serotonin also may mediate and moderate long-term deleterious effects of early stress and anxiety on adult outcome. Thus, treating developmentally appropriate anxiety unnecessarily by manipulating serotonin carries risks; nevertheless, failing to address an underlying serotonin dysfunction may also carry risk. Inconsistent data in rodents and nonhuman primates force treatment decisions to remain grounded in clinical trials with humans. As with CBT, long-term data justify use of SSRIs for at least 1 year, particularly if anxiety recurs following discontinuation of the SSRI (Pine, 2002). Nevertheless, no randomized controlled trials compare sustained relative to discontinued long-term SSRI treatment in pediatric anxiety. As such, more research on the risks and benefits of chronic treatment is needed.

\section{ATTENTION BIAS}

\section{Clinical Correlation}

Both SSRIs and CBT are thought to induce clinically meaningful change by balancing perturbed neural and cognitive processes associated with threat perception and interpretation. Such therapies may target specific biases in threat processing, such as hypervigilance toward threats, emerging in specific social evaluative contexts, or they may target more general processes, such as those that engender rumination on threats associated with excessive worrying. Insights on novel therapeutics might emerge by focusing specifically on the manner in which CBT and SSRIs alter specific processes, such as attention bias toward threat.

Increased attention to threat serves an adaptive function by facilitating the detection of danger (LeDoux, 2000). Advantaged allocation of processing resources to overt threats is adaptive, in that it facilitates responses to dangerous situations. However, biased threat processing has been implicated in the etiology and maintenance of anxiety disorders (Bar-Haim et al, 2007; Beck, 1976; MacLeod et al, 2002; Williams et al, 1996). Indeed, considerable research demonstrates that the attention system of anxious individuals is distinctively sensitive to threats (Bar-Haim et al, 2007; Mogg and Bradley, 1998). Thus, allocating attention toward extreme threat represents a normative response; however, a reduced threshold for eliciting threat-related attention allocation to mild threats represents a core feature of clinically significant anxiety.

Various paradigms quantify threat-related attention biases, each showing replicable effects. Nevertheless, a focused review on specific attention-related behaviors may clarify key aspects of complex relations among attention, threat-processing, and anxiety, manifest across development and even across species. When considering a focused perspective, the dot-probe paradigm probably represents the single best task affording a developmental perspective (MacLeod et al, 1986; Pine, 2007). This task assesses individual differences in attention-orienting behavior to one or another spatial location, which occurs in the context of a target-probe identification paradigm. On each trial (Figure 1), a central fixation cross is displayed, followed by presentation in opposite hemifields of two stimuli, one threat related (eg, angry face) and one neutral (eg, neutral face). Upon offset, a target probe appears at the location occupied by one face, which participants detect as quickly and accurately as possible. Response latencies provide a 'snapshot' of attention allocation with fast responses occurring to probes at attended locations. Manipulating timing of face-probe offset-onset presentations shifts the 'snapshot' timing. Thus, chronometry of threat-attention interactions is examined by measuring bias across various timing parameters.

Meta analysis (Bar-Haim et al, 2007; Williams et al, 1996) documents a moderate effect size of attention bias across diverse anxious groups. As such, attention bias represents a general feature of between-group differences in anxiety, and the bias is not observed typically in nonanxious individuals. Threat bias represents an instance where attention prioritization of low-level threat-related material occurs only in specific, anxious subgroups but not in most healthy individuals. Quantitative review also suggests that relatively 
a Events in dot probe experimental trials

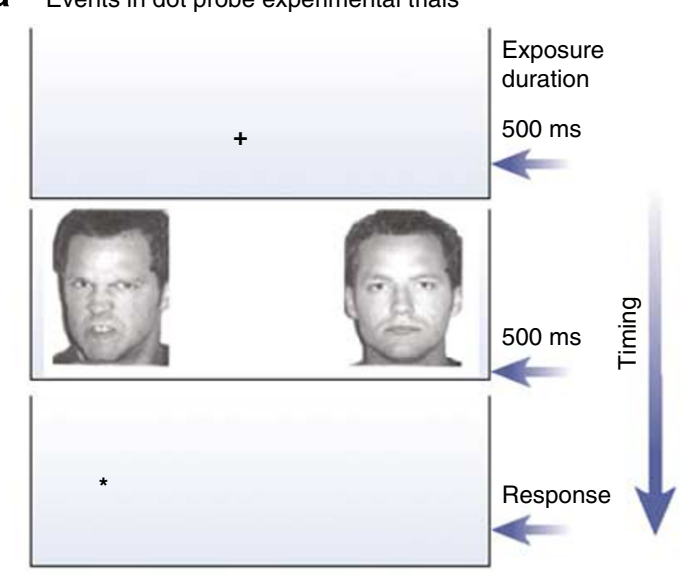

c

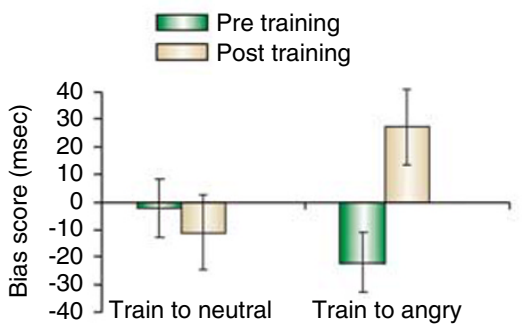

Increased attention toward threat in nonanxious children following training to attend to angry faces (left); Increased anxiety in these children following stress induction.

b

Events in a training experiment - testing for causality of threat bias in anxiety

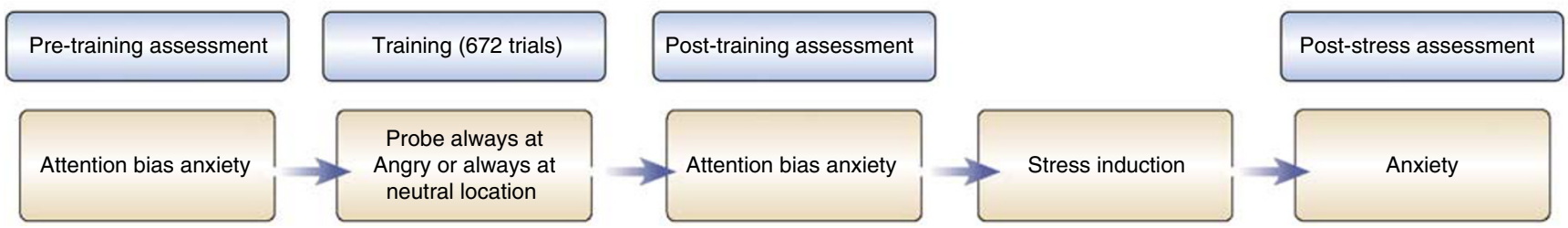

Figure 1. The dot-probe paradigm. (a) The structure of the dot-probe paradigm. Trials begin with fixation and end with an asterisk probe. These events are separated by a face pair, which can vary across experimental trials in terms of depicted emotions and the timing. Hence, faces can include an angry and neutral picture, or different emotions, each presented for varying times, including $500 \mathrm{~ms}$ as shown, or other times, such as brief exposures (eg, $17 \mathrm{~ms}$ with a mask). (b) The design used in a dot-probe training experiment with children, as described in more detail in Eldar et al (2008). This experiment involved an initial pretraining assessment, a training session designed to induce attention bias, a posttraining reassessment, and a stress induction paradigm. (c) Summary of results from Eldar et al (2008). Bias training successfully induced greater attention bias and anxiety responses to stress than a control training regimen.

slow-developing, elaborative processing contributes to threat biases. However, on the dot-probe task, a significant bias is also observed with brief (eg, $17 \mathrm{~ms}$ ) threats, stimuli that are difficult, if not impossible, to consciously perceive. Moreover, subliminal exposure to threat-neutral displays in anxious, relative to nonanxious individuals, yields an effect size almost twice as large as for supraliminal exposures (Bar-Haim et al, 2007). This implicates early, rapidly and implicitly deployed, attention processes in individual differences.

\section{Development}

Work has begun to examine the threat bias/anxiety association from a developmental perspective. This is important, given the strength of findings in adults, coupled with other data on developmental aspects of clinical anxiety (Pine, 2007). The extant literature establishes that children as young as 7 reliably perform the dot-probe task.

In terms of attention bias's role in early anxiety, three positions have been advanced. One suggests that threatrelated attention biases and individual differences in anxiety go hand in hand from early in development (Martin et al, 1992). That is, an association between threat bias and individual differences in anxiety might emerge even in preschoolers, considering individual differences in anxiety manifest either as clinical pathology or as associated potential precursors, such as behavioral inhibition (Pine, 2007). A second position (Kindt et al, 1997) suggests that the association between threat bias and between-group differences in anxiety only manifests relatively late in development. That is, both anxious and nonanxious children are presumed to enter early development with an equally strong bias toward threat, a bias not found in nonanxious adults. With increasing age, nonanxious children selectively learn to inhibit this bias, whereas anxious children fail to do so. Finally, a third position (Fox et al, 2007) suggests that attention bias is shaped by interactions between temperament and caregiver behaviors. Certain children, predisposed to fear novelty, may be raised in ways that heightens attention to threats, thereby contributing to eventual onset of clinically significant anxiety. Of note, all three theories may be compatible with observations associating ventral prefrontal cortex (vPFC) maturation with increased modulation of subcortical structures in which fear reactions are represented.

For the first position, individual differences are expected early, such that only anxious infants and young children demonstrate attention biases. Individual differences in vPFC maturation might ultimately account for the fact that many of these anxious children mature to become nonanxious adolescents and adults. As such, processes whereby vPFC exerts increasing control over amygdala engagement may be analogous to extinction of fears, but 
played out over the extended time course of development as opposed to relatively narrow window of fear-conditioning experiments (Quirk and Gehlert, 2003). For the second position, attention bias is expected in both anxious and nonanxious infants and young children (Kindt et al, 1997). Here, prefrontal cortex (PFC) maturation might reduce these biases only in those individuals who remain nonanxious. This position receives partial support from work finding threat bias in both anxious and nonanxious children. By contrast, other studies find a significant bias only in anxious children (Waters et al, 2008). For the third position, attention biases are expected to emerge early in the unique subset of children born with an underlying anxiety predisposition and exposed to particular rearing environments. Here, interactions between predispositions and rearing are expected to sculpt behavior through their evolving effects on vPFC-amygdala circuitry development.

Thus, the first and the third positions predict threat bias in particular subsets of at-risk or affected children, whereas the second position predicts threat bias in most, if not all children. These discrepancies might be resolved through studies that rely on longitudinal designs measuring threat bias very early in life in at-risk youths. For example, a study following at-risk offspring of anxious parents might clarify the degree to which individual differences in threat bias predate or reflect later anxiety. Moreover, by examining the influence of both genetic predisposition and caregiver behavior, such a study might clarify the degree to which environmental influences interact with early-manifesting risk to shape both attention bias and clinical anxiety.

\section{Cause}

Attention bias probably represents the single most frequently noted correlate of individual differences in anxiety. Nevertheless, scant empirical evidence clarifies the degree to which attention bias represents a downstream effect, as opposed to a causal factor. Experimental manipulations provide one method for evaluating causality, whereby training procedures induce attention bias, followed by an assessment of stress reactivity. In two studies, MacLeod and colleagues (MacLeod et al, 2002) were the first to causally implicate training-induced attention biases in stress reactivity. Specifically, in these studies, nonanxious adults were randomly assigned to training conditions. One condition induced bias toward threat, by repeatedly pairing target probes with the spatial location of threat cues. The other induced avoidance by pairing probes to the location of the neutral stimuli. Experimentally induced bias did not affect anxiety following training but it did cause congruent changes in state anxiety following a stress induction task.

A growing number of experiments document reliable effects of training on attention bias (Clarke, 2008; Dandeneau et al, 2007; Eldar et al, 2008; Wilson et al, 2006). Of note, none of these studies utilizes very brief threat exposures, so effects only pertain to stimuli that can be readily perceived. Regardless, by reliably demonstrating robust training effects, the work shows that attention bias is not strongly trait-like. Of particular relevance for the present review, one such study trained children to have a bias (see Figure 1; Eldar et al, 2008). This study showed that attention bias influenced children's response to an acute laboratory stressor. Thus, attention biases can be reliably manipulated in the laboratory. This work sets the stage for the first set of future studies that eventually will evaluate the clinical relevance of these training effects.

\section{Treatment}

A particularly important next step will be to explore the role of computer-based attention training in anxiety disorder treatment. No published studies consider this issue. Evidence of clinically relevant effects in patients could directly link research on therapeutics and attention bias. For example, for some children, computer-based training of attention may be more acceptable than traditional in-person therapy formats, and it may offer advantages by delivering exposure where attention can be controlled in a systemized manner. Perhaps most importantly, such computer-based approaches may augment the approach to attention retraining that forms a core feature of CBT. With CBT, patients attempt to use cognitive control strategies to willfully alter their attention focus. With computer training, subjects are taught implicitly to control attention, through repetitive training procedures, in the absence of explicit instructions. Thus, combining CBT with computerized retraining may simultaneously target explicit and implicit processes. As such, computer-based interventions have potential for improving outcomes in research on child anxiety disorder therapeutics.

\section{Neural Circuits}

Consistent observations linking anxiety to threat bias suggest the importance of examining associated neural circuitry. Demonstrating parallel developmental relations across species among threat bias, anxiety, and neural architecture would increase the clinical relevance of research in animals and possibly inform therapeutics. Such parallels are expected, given other evidence of cross-species conservation in the neural architecture of fear.

Research on the rodent threat response precisely delineates the topography and chronometry of distributed neural circuitry engagement during threat-orienting behavior. Engagement of the amygdala, immediately following threat exposure, represents a key early-occurring process initiated for visual threats when information passes from the retina to the thalamus and immediately to the amygdala (LeDoux, 2000). This process is thought to provide very quick but relatively crude signals to the organism, associated with engagement of attention in the service of early threat detection and stimulus reinforcement learning (Blair et al, 2005). As such, this early process reflects the central role of 
the amygdala in immediate, rapid, implicit modulation of attention orienting to threats (Davis and Whalen, 2001). Data in humans demonstrate comparable effects (Phelps and LeDoux, 2005). Accordingly, from the clinical perspective, this view suggests that hypersensitivity in the amygdala may be involved in anxiety-related perturbations manifest in the immediate response to threats.

Following quick amygdala engagement, research in rodents demonstrates more gradual cortical engagement, providing more detailed representation of threat features (LeDoux, 2000). Such detailed representation facilitates engagement of architecturally more complex cortically based processes, which also allow flexible modulation of amygdala-based processes. Other work implicates vPFC afferents specifically in modulation of amygdala engagement (Quirk and Mueller, 2008). From the clinical perspective, this suggests that healthy vPFC engagement, when encountering threats, represents an adaptive phenomenon. This might counteract a diathesis, manifest in amygdala hypersensitivity, which allows pediatric anxiety to remit. Conversely, clinical expression may reflect failure of vPFC maturation to counteract an underlying diathesis.

Available imaging data using variants of the dot-probe task in youths (Monk et al, 2006; Monk et al, 2008) demonstrate the relevance of data on topography and chronometry of fear circuitry function in rodents (Figure 2). Thus, exposure to very brief threats in the context of a dotprobe experiment produces amygdala engagement in anxious but not healthy adolescents (Monk et al, 2008). The magnitude of this engagement positively correlates with both anxiety severity and attention bias, consistent with the view of early amygdala engagement as representing anxiety and associated threat bias. Activity in vPFC, in contrast, correlates negatively with activity in the amygdala during

a

Events in experimental trials

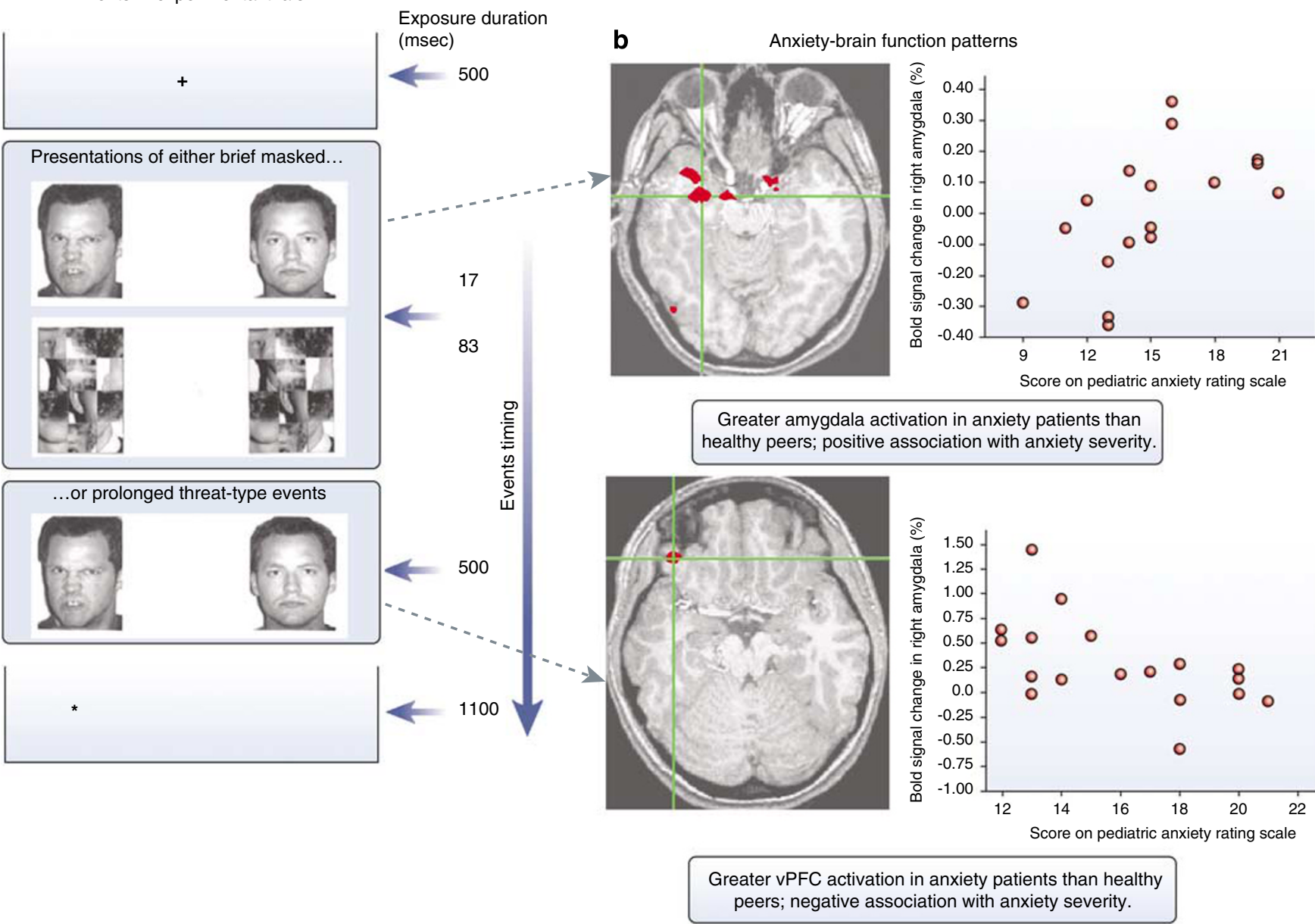

Figure 2. Two fMRI studies of amygdala-prefrontal cortex (PFC) engagement in adolescent generalized anxiety disorder (GAD). (a) Stimulus event timing parameters in the two studies. For both, stimuli began with a $500 \mathrm{~ms}$ fixation symbol and ended with an $1100 \mathrm{~ms}$ asterisk dot-probe target. However, for facial stimuli, one study utilized brief $17 \mathrm{~ms}$ faces, followed by an $83 \mathrm{~ms}$ backward mask; the other study utilized $500 \mathrm{~ms}$ faces with no mask. (b) Results. The top of Panel b shows the location of a between-group right amygdala difference where GAD showed greater activation than healthy adolescents in a masked $17 \mathrm{~ms}$ threat-vs-neutral-face contrast. The figure also shows a positive correlation between anxiety severity in GAD and amygdala engagement. The bottom of Panel b shows the location of a between-group difference in right PFC activation where GAD showed greater activation than healthy adolescents in an unmasked $500 \mathrm{~ms}$ threat-vs-neutral contrast. The figure also shows a negative correlation between anxiety severity in GAD and PFC engagement. More details of methods and results from these studies appear in Monk et al (2006, 2008). 
brief threat exposure, with stronger correlations in healthy than anxious adolescents. This suggests that perturbed interactions between $\mathrm{VPFC}$ and the amygdala support attention bias and the clinical state. Data from a second dot-probe experiment, using longer threat exposures, also support this view (Monk et al, 2006). Here, data demonstrate increased $\mathrm{VPFC}$ engagement in anxious relative to healthy adolescents, with no between-group differences in the amygdala. This suggests that $\mathrm{vPFC}$ engagement during longer threat exposure reflects the influences of cortically based regulatory processes over underlying hypersensitivity, presumably involving the amygdala and associated subcortical structures. Consistent with this view and in contrast to data during brief threat exposure, vPFC engagement with longer exposures predicts lower levels of anxiety among anxious youths. Thus, data in rodents and humans implicate the amygdala in initial threat reactions and PFC-amygdala connectivity in later regulatory responses. Of note, however, key questions emerge. For example, imaging studies most consistently implicate lateral vPFC in threat-attention interactions, where data on extinction most consistently implicate medial PFC (mPFC). This raises questions addressable through research on animals about the nature of developmental relations among amygdala and specific PFC-based functions.

\section{Future Studies}

Research in nonhuman primates could facilitate work on therapeutics. As a first step, working with Jim Winslow, we have begun to examine the effects of threat on orienting in juvenile nonhuman primates, through an examination of eye-scanning patterns exhibited in a variant of the dotprobe task. This work fits within a broader context relating human and nonhuman primate development to diverse indicators of anxious behavior, including facial expressivity and vocalization (Nelson et al, 2002). As with research on cognition, a narrow focus may provide traction when attempting to bridge data across development and across species. Thus, work focused narrowly on the dot-probe task models specific, highly salient behavior, to facilitate a mechanistic understanding of specific cross-species parallels. This narrow focus, however, serves to bridge broader research demonstrating the manner in which anxious behavior exhibits parallel changes across development in a range of mammalian species.

As shown in Figure 3, juvenile nonhuman primates exhibit a tendency to orient selectively to threat stimuli. As a second step, in ongoing work, we have begun to examine the relationship between individual differences in anxious behavior and threat orienting. The demonstration of distinct threat-orienting behavior in anxious relative to a

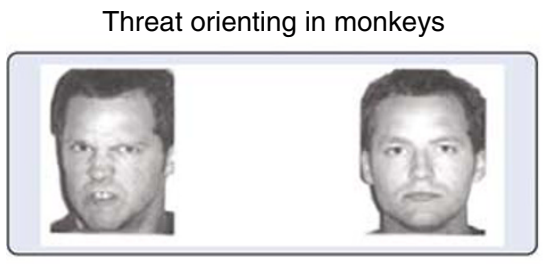

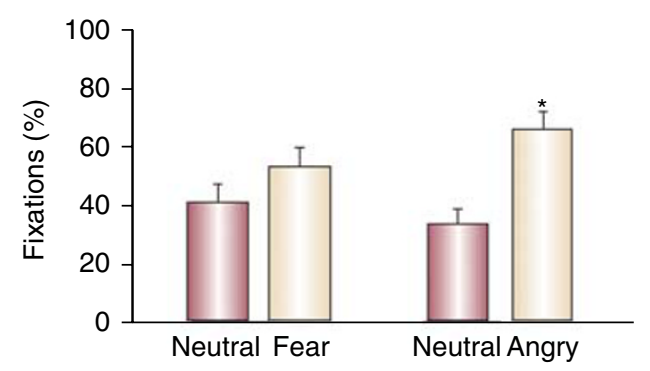

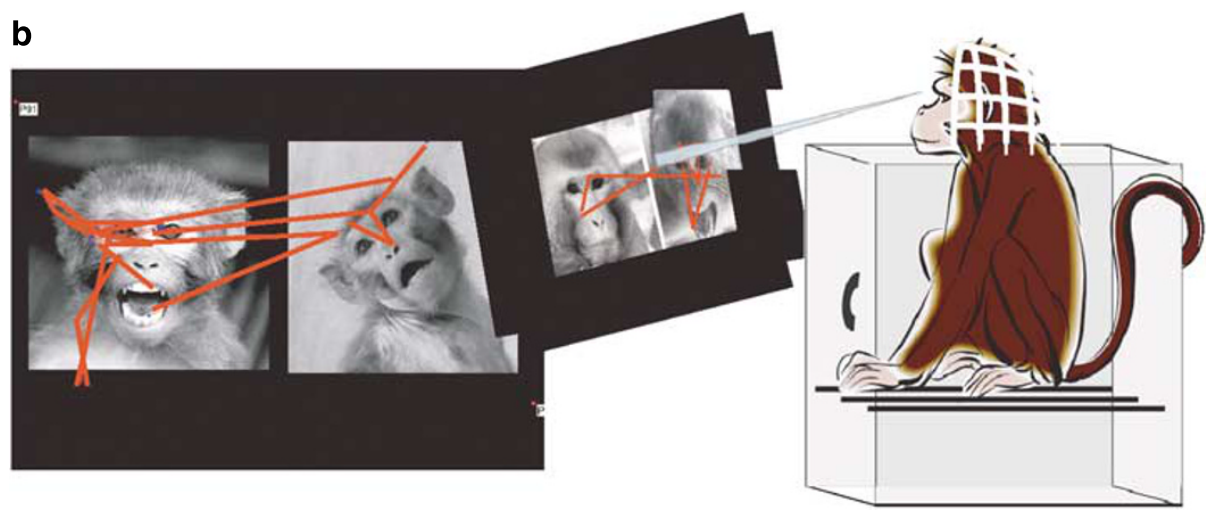

Figure 3. Procedures for monitoring eye tracking in adolescent rhesus monkeys. These procedures involve displaying photographs while monkeys' gaze fixations are monitored with an infrared camera. (a) Data in four monkeys during the viewing of human threat-neutral faces. Panel a shows an example of utilized stimuli, as also employed in dot-probe studies with human adolescents. The adjacent graph depicts the mean percentage of gazes to human faces in neutral-fearful pairs (left) and neutral-angry pairs (right). A paired $t$-test of fixation percentages to neutral vs angry expressions revealed a significant difference in orientating toward angry expressions, paired $t=2.3, p<0.05$. Thus, monkeys spend significantly more time viewing threat relative to neutral faces as depicted during the dot-probe task. (b) A schematic representation of the apparatus, where a monkey is seated in a primate chair while pairs of photographs are presented on a computer. Although pilot work used human faces, ongoing work is using monkey faces, as shown in the figure, to increase ecological validity. An example of gaze fixation patterns from one monkey to a typical face pairing is displayed on the left. 
nonanxious monkeys would further suggest the suitability of modeling threat-orienting behavior in young monkeys to facilitate understanding of pediatric anxiety. Third, demonstrating a parallel affect of SSRIs on threat-orienting behavior in monkeys and children would further solidify the suitability of research on threat-orienting behavior in nonhuman primates as a model for threat biases in pediatric anxiety disorders. Establishing primate attention training paradigms might also facilitate research on the degree to which attention-related plasticity extends across contexts. Thus, imaging studies relate individual difference in brain function to individual difference in attention bias, supporting the contention that individual differences in anxiety relate to difference in brain development. However, attention bias can be trained rapidly, showing an influence operating on very brief timescales. Studies in nonhuman primates might reveal the manner in which these brief training effects interact with brain development to shape behavior.

Following demonstration of such parallels, research on nonhuman primates could stimulate research on therapeutics. Such an approach could involve procedures, such as invasive imaging methods, reversible lesions, or intracellular recordings, which precisely elucidate the role of amygdala-vPFC circuitry in threat-orienting behavior. Moreover, novel therapies could be evaluated based on their capacity to alter threat-orienting behavior through direct effects on circuitry. Demonstrating robust effects on behavior would provide further justification for developing suitable procedures for safely altering threat-orienting behavior in humans.

\section{LEARNING ABOUT DANGER \\ Overview}

Research on attention bias facilitates basic neuroscience extension of clinical work; research on processes engaged when organisms learn about danger facilitates clinical extension of basic work. Circuitry engaged in these instances shows strong conservation across phylogeny, facilitating basic clinical integration. Functions represented within this circuitry can be parsed into four processes: (1) learning to associate a discrete neutral stimulus with an aversive event that immediately succeeds it (ie, 'cue conditioning'); (2) conditioning to context, defined as learning to associate fear with contextual cues present in the environment (ie, 'context conditioning'); (3) consolidating/reconsolidating learning, defined as transferring learned information into long-term memory after its acquisition or retrieval (ie, 'reconsolidation'); and (4) extinction of fear responses, defined as learning to no longer respond to a previously conditioned stimulus (CS) or context after it has been presented without the aversive event (ie, 'extinction'). The ability to perform each task requires dissociable neural pathways that develop on different timescales both across ontogeny and within the learning environment.
Therapeutic insights emerge from understanding the manner in which individual differences in learning developmentally shape individual differences in anxiety. As such, this section begins by reviewing developmental data on these four learning processes. This is followed by a brief review of data in basic science on the manner in which learning gives rise to individual differences, with a focus on therapeutics. Finally, data are reviewed from initial efforts to extend basic findings to the clinic, particularly through treatment-related research.

\section{Development and Learning About Danger}

Cue conditioning. Classical conditioning represents the best-understood fear-learning process (LeDoux, 2000). It produces synaptic changes in lateral amygdala (LA) neurons, where two pathways coincide: one from the thalamus and sensory cortex transmitting information about the CS and a second transmitting information about the unconditioned stimulus (US). LA cells change their responses to the CS after CS-US pairing, presumably via long-term potentiation (LTP; Rogan et al, 1997). LTP can be divided into two parts: 'early' LTP (E-LTP), which is thought to produce short-term acquisition, and 'late' LTP (L-LTP), which is thought to support consolidation into long-term memory. Although E-LTP involves protein phosphorylation (Schafe et al, 2001), L-LTP involves protein synthesis (Malenka and Nicoll, 1999). LTP can be associative, in that strong activation at one synapse potentiates other weakly activated synapses (Malenka and Nicoll, 1999). Associative properties may result from synaptic tagging, where stimulation in one synapse attracts proteins required for the induction of L-LTP. This allows other synapses on the cell to be potentiated, so long as the one synapse has been stimulated sufficiently to induce LTP (Dudai, 2006; Frey and Morris, 1997). In this way, LTP can lead to the strengthening of CS input synapses after activation of a target cell by US inputs.

Work in rodents finds that early-emerging conditioning responses involve immature forms of cue-specific learning that disappear and are replaced by mature forms. Thus, in terms of early learning, rats display unique conditioning, characterized by failure to engage the amygdala in fear (odor-shock) conditioning before postnatal day (PND) 9, although the incorporation of the amygdala into odormalaise learning occurs closer to weaning (PND 22-23; Sevelinges et al, 2007; Shionoya et al, 2007; Shionoya et al, 2006; Sullivan et al, 2006). Interestingly, although more mature rats do not display these immature forms of amygdala-independent learning, they do manifest the lasting effects of early-life conditioning (Sevelinges et al, 2007). These data are consistent with other work demonstrating how the uniquely plastic state of the immature fear circuit establishes life-long patterns of fear-related behavior (Meaney, 2001). Furthermore, as rodents continue to develop, learned behaviors become more complex as the infant's behavioral repertoire incorporates more adult-like patterns of fear expression (Hunt and Campbell, 1997). For example, other components of a mature fear response, such 
as potentiated startle, emerge as output pathways continue to mature (Hunt and Campbell, 1997). Interestingly, studies in mice demonstrate further refinements in cue-specific conditioning across adolescence (Hefner and Holmes, 2007). Thus, categorically distinct, qualitatively immature forms of conditioning operate transiently in early life, such that immature organisms exhibit threat-response behavior in ways not shown by older organisms. When these forms disappear, they are replaced in adolescence by more mature forms, which then change in an incremental fashion.

Context conditioning. Context conditioning differs from cue conditioning. The two processes rely on separable neural architecture engaged in distinct developmental timescales. When organisms develop conditioned responses to discrete cues, they also condition to components of the environment in which cues are embedded, such as patterns on the walls of the training box. Because contexts are present before, during, and after CS-US pairings, contexts, relative to cues, more ambiguously predict aversive events. Contexts alert animals to impending danger but provide imprecise information about timing or location. Thus, cue conditioning might index fear, whereas context conditioning might index anxiety (Davis, 1998).

Given that close CS-US temporal proximity facilitates LTP, it is unsurprising that cue conditioning involves different architecture than context conditioning, which appears to require the hippocampus as well as amygdala subnuclei distinct from those involved in cue conditioning (Kim and Fanselow, 1992; Phillips and LeDoux, 1992). However, distinct inputs for cues and contexts project to the same outputs (LeDoux, 2000), which is thought to explain the qualitatively similar appearance of fear responses to cues and contexts. Thus, although the nature of fear learning to cues can be differentiated from learning to contexts, once acquired, the nature of the fear response does not differ. Conditioning to cues and contexts can also be dissociated from a developmental perspective. Although rats freeze to conditioned cues by PND 10, freezing to conditioned contexts appears later, roughly around weaning, PND 21 (Rudy, 1993).

Viewed broadly, these observations carry developmental implications: they suggest that fears apparently involving similar behavioral responses can reflect clearly dissociable underlying neural architecture. Nevertheless, viewed more narrowly, the precise manner in which clinical phenomena reflect trends observable in data on rodent cue and context conditioning remains unknown. Given the many differences between basic and clinical research, direct applications of basic work to the clinic require research examining the relationship that cue and context conditioning, studied directly in children, show with specific clinical manifestations.

Consolidation. Consolidation involves the transfer of knowledge into long-term memory, presumably through L-LTP in the neural circuit in which CS-US associations are stored. L-LTP requires protein synthesis in the postsynaptic cell, which can change the postsynaptic neuron indefinitely
(Schafe et al, 2001). The amygdala but not hippocampus is required for consolidation of CS-US associations in cue conditioning (Nader et al, 2000); the hippocampus is required to consolidate context-US associations (Kim and Fanselow, 1992). Components of the molecular cascades engaged by these processes have been precisely delineated (Fischer et al, 2004; Vianna et al, 2003). Data on development of consolidation parallel data on conditioning to cues and contexts. Thus, rats can consolidate conditioning for discrete cues by PND 18 but cannot consolidate conditioning about contexts until PND 23.

Each time a CS-US association is retrieved, the trace becomes labile and requires 'reconsolidation' (Nader et al, 2000). This provides an opportunity for new information about the circumstances of retrieval to be incorporated into the trace (Dudai, 2006). This process can recur over time (Schmidt and Bjork, 1992), such that new information can be added to each retrieved memory, providing more socalled 'hooks' for distinct recollections. Developmentally, this process might impact the initiation and maintenance of individual differences. If individuals have an initial disposition to more readily attend to, retrieve, and ruminate on conditioned fear memories, each retrieval event could lead to reconsolidation that facilitates future retrievals and prevents the memory from 'fading' as it might otherwise do. Such repeated recollections would serve to strengthen fear memories and broaden the cues that elicit them.

Extinction. Extinction allows the organism to refrain from exhibiting fear after a CS has been unpaired with the US. Extinction involves new learning, as opposed to erasing the CS-US association, as evidenced by data on renewal, reinstatement, and related processes (Bouton, 2002). As such, extinction can be differentiated from forgetting or failures to reconsolidate, which involve erasure (Duvarci and Nader, 2004). Extinction has been viewed as a form of learning that encodes contexts in a similar fashion as context conditioning but on a temporal as opposed to spatial dimension. As such, extinction allows the organism to deploy fear responses flexibly, eliciting responses appropriate at some time points but not others (Bouton, 2002).

Extinction requires the amygdala, hippocampus, and the mPFC specifically, the infralimbic cortex (IL) with each region mediating a specific process. Blockade of LA glutamate receptors associated with E-LTP impairs acquisition of extinction (Sotres-Bayon et al, 2007), whereas blockade of hippocampal L-LTP prevents consolidation of context conditioning extinction (Quirk and Mueller, 2008). The mPFC is critical in extinction, through interactions with the amygdala and hippocampus. For example, extinction requires IL cortex engagement during processing of an extinguished CS. As the PFC develops relatively late, one also would expect extinction to be a rather late-developing skill. Although rats as young as PND 17 can exhibit withinsession extinction (Carew and Rudy, 1991), the ability to consolidate this information across sessions matures later. Recent evidence (Kim and Richardson, 2008) suggests that 
extinction erases the initial fear memory in PND 16 rats, unlike in mature rats. Maturation in mPFC-hippocampal interactions likely contribute to these changes. For example, extinction is sensitive to context: a CS, conditioned in one context but extinguished in another, elicits the original fear response in the initial context. This hippocampally mediated process matures in the rat between PNDs 17 and 21 (Carew and Rudy, 1991). Finally, as reviewed below, considerable interest in clinical extensions of basic work follows from these conceptualizations of extinction.

\section{Individual Differences}

Basic research generates two key insights on relations between learning and individual differences. First, in a developmental context, an anxious phenotype arises in tandem with signs of enhanced fear conditioning to ambiguous information - particularly, a tendency to exhibit fear behaviors in the presence of ambiguous cues. Second, individual differences arise through interactions between experiences and genes.

Work on the serotonin receptor 1A knockout (5HT1AR$\mathrm{KO})$ mouse implicates genes in anxiety and also provides a sound model for studying individual differences. This mouse exhibits normal cue-specific fear conditioning, in tandem with a specific perturbation in context conditioning that leads the mouse to show similarly heightened fear responses to both a conditioned context and an ambiguous context that shares some, but not all, features with the conditioned context (Klemenhagen et al, 2006). Wild-type mice, by contrast, show some fear response to the ambiguous environment, but a distinctly smaller response than that elicited by the conditioned environment. Thus, the deficit in the 5HT1ARKO mouse involves a perturbation in the ability to discriminate cues in terms of the level of danger they convey. This deficit is mediated by changes in the hippocampal dentate gyrus, a region critical for context conditioning, a process that emerges during a specific phase in development (Tsetsenis et al, 2007).

Importantly, because factors influencing the 5HT1ARKO phenotype can be pinpointed both anatomically and temporally, research on this mouse generates specific questions. Altering forebrain serotonin function only from PND 4 to PND 21, a crucial time for hippocampal development, generates the 5HT1ARKO phenotype, both for ambiguous context conditioning and broader behaviors. This raises questions about the manner in which human anxiety reflects the temporally specific influence of serotonin on amygdala-hippocampal development. Although work is only beginning to emerge, studies in rodents also show that early-life stress interacts with manipulations of serotonin-related genes (Carola et al, 2008). This, in turn, raises specific questions on the manner in which early-life stress in the human interacts with serotonin and brain development. More work is needed, however, examining such issues directly in children. The marked differences between rodents and humans, both in terms of neural architecture and brain development, preclude precise oneto-one cross-species mapping of serotonin-related events.

Despite cross-species differences, data do broadly suggest that inability to discriminate between overtly dangerous and ambiguous cues contributes to an anxious phenotype. Much like attention bias in humans, perturbed ambiguous cue processing in mice represents an association between a broad, anxiety-related individual difference profile and a lowered threshold for eliciting fear responses in specific situations that appear only minimally threatening. Thus, as in the dot-probe task, when presented with a stimulus containing both threats and safety cues, 5HT1ARKO mice respond to the threats more than wild-type mice. Likewise, anxious humans, when presented with both threatening and nonthreatening information, attend to the threat cues.

Beyond conditioning's role in shaping individual differences, data on early-life rearing manipulations raise broader questions on the role of experience. Research in this area reveals profound and highly stable individual differences in anxious behavior following environmental manipulations that alter the expression of certain brain proteins. Specifically, within a rodent strain, experimental rearing manipulations produce robust neural and behavioral individual differences (Meaney, 2001). Similarly, across rodent strains, individual differences in anxious behavior and associated neural functions partially reflect individual differences in the rearing environment (Caldji et al, 2004). These relationships emerge through effects on neural architecture previously implicated in conditioning and extinction. Specifically, effects of early-care manipulations on anxiety involve alterations in $\gamma$-aminobutyric acid (GABA)-A subunit expression in mPFC, hippocampus, and amygdala (Caldji et al, 2004).

These effects change behaviors through the influences of experience. If learning can be defined broadly as the effects of experience on behavior, then rearing effects in rodents can be viewed as a form of learning that shapes individual differences. However, available research has not elucidated the manner in which rearing shapes behavior through effects on specific information-processing functions previous linked to fear learning, such as conditioning and extinction. Of note, given a role for $\mathrm{mPFC}$, hippocampus, and amygdala-based GABAergic pathways in extinction learning, one might expect rearing to influence anxious behavior through effects on conditioning or extinction.

\section{Clinical Translation}

Clinical attempts to translate basic findings have pursued two complementary avenues. First, considerable research examines how individual differences in fear conditioning relate to individual differences in anxiety. Second, considerable research attempts to use novel treatments derived from basic research on fear learning. Of note, as with research on SSRI treatment in children, research in both areas faces considerable challenges. Thus, studies of fear conditioning must contend with the ethical constraints that 
confront attempts to study children's responses to aversive scenarios (Lau et al, 2008). Similarly, studies of novel therapeutics must contend with concerns about exposing children to new therapies before efficacy and safety has been established in adults (Fost, 2001). As a result, the following sections review work on individual differences and on treatment, emerging largely from work in adults, while emphasizing findings from the few studies in children and adolescents.

Individual differences. A long tradition considers the role of conditioning and extinction in human anxiety. Despite this history, the nature of relations between conditioning or extinction and individual differences remains poorly understood. A recent meta-analysis demonstrates the incorrectness of many prior theories (Lissek et al, 2005): in the classic discriminative fear-conditioning experiment, individual differences in anxiety show no relationship to individual differences in conditioning or extinction; such relationships only emerge on simple conditioning paradigms. Much as in the 5HT1ARKO mouse, data in both adults and children suggest that conditioning and extinction-related deficits reflect failure to discriminate among overtly dangerous and ambiguous cues. This accounts for observations of enhanced conditioning in anxiety disorders on simple as opposed to discriminative conditioning experiments (Lau et al, 2008; Lissek et al, 2005). The failure to observe between-group differences in discriminative paradigms reflects the enhanced responses in patients both to threat and safety cues, findings receiving admittedly equivocal support in adolescents (Lau et al, 2008). As discussed below, these data generate specific hypotheses on novel treatments.

In terms of specificity, data suggest that individual differences emerge for an isolated set of disorder-relevant conditioning-related experiences. Data in rodents suggest that mature conditioning responses to specific situation, such as discrete cues as opposed to contexts, emerge at different points in development. In terms of human development, considerable work documents parallel dissociations, where distinct forms of human fear show unique developmental profiles (Ollendick et al, 1996).

Perhaps the strongest clinically relevant data on specificity in conditioning-related experiences emerge for studies of social fears. Thus, although anxiety disorders, in general, show normal fear conditioning to disorder-irrelevant cues, social phobia, specifically, involves perturbed conditioning to aversive social experiences (Lissek et al, 2008b). Figure 4 schematically depicts an aversive social conditioning experiment with which this association has been demonstrated. Although the actual experiment used adult photographs, the schematic figure employs adolescent photographs to emphasize the relevance of the paradigm for developmental views on social fear. Developmental relevance emerges from the fact that adult social phobia virtually always arises against a background of prior childhood or adolescent fearfulness. Research on the relations between early-childhood temperament and later-life social fears further implicates life-long patterns of social learning in individual differences in social anxiety.

Data on fear learning generate a framework for conceptualizing how early predispositions develop into later behavioral inhibition and adult social phobia. Of note, adult social phobia is likely to arise from multiple pathways, an example of 'equifinality'. Data on fear learning suggest just one potential pathway. This pathway begins with the observation that some infants display hypersensitivity to novelty, presumed to reflect hyperexcitability of brain structures that detect threat (eg, amygdala). These individual differences may provide the context in which minor cues present in aversive social encounters come to activate the fear system and capture attention. Such minor cues are typically ignored by others, but they act as a CS in hyperreactive infants and toddlers. Many such social encounters across multiple contexts will lead to repeated reconsolidation of the fear response, lowering the threshold for social encounters to elicit anxiety. These encounters enable reconsolidation of fear learning during early childhood.

Adolescence and increasing maturity of vPFC brings new opportunities for extinction to influence early-emerging individual differences. However, by the time maturing vPFC processes support extinction, fear learning may have occurred across multiple contexts that also must be extinguished across multiple contexts (Bouton, 2002). As a result, extinction would require pervasive and repeated exposure to change an established network of associations. Social fears would only resolve in the subset of adolescents who can sustain these necessary vPFC-based processes, either through their unique experiences or intrinsic aspects of their circuitry. Social fears would be expected to evolve into chronic mood and anxiety disorders among those adolescents who lack this capacity.

This framework suggests that perturbed attention orienting and fear learning occurs primarily in the subset of inhibited children who go on to manifest clinically significant anxiety as adolescents and adults. If these predictions are confirmed, the framework would carry direct implications for early identification, prevention, and treatment. Translating these implications to the clinic, however, would require far more work on the precise manner in which brain development influences specific treatment decisions, be they pharmacological or psychological.

Neuroimaging studies that adapt the paradigm in Figure 4 could test the accuracy of this framework while generating data on underlying neural architecture. In doing so, such work provides an important avenue for demonstrating cross-species parallels. Figure 4 schematically illustrates aspects of the above-noted temperament-based framework, extending data in rodents and nonhuman primates. This work generates the specific hypothesis that individual differences in conditioning-related learning in social phobia reflect early-emerging individual differences in amygdala function and their regulation during adolescence, when vPFC function evolves. If neuroimaging studies could confirm this hypothesis, these data would provide insights 
a

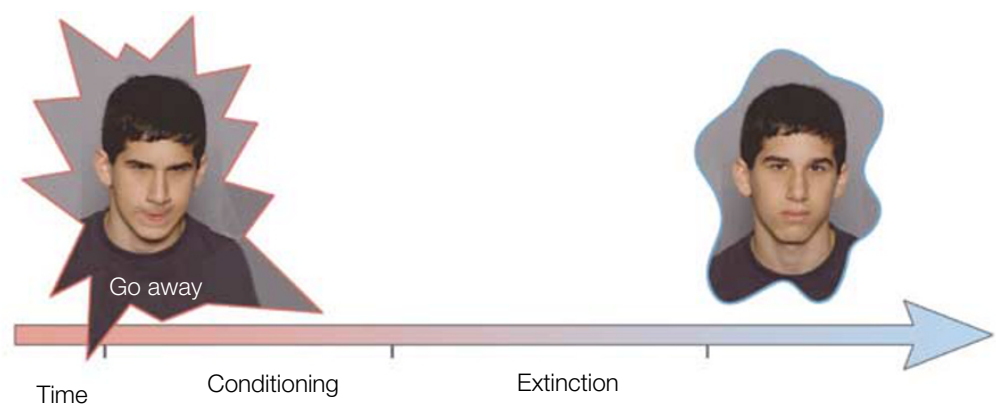

b
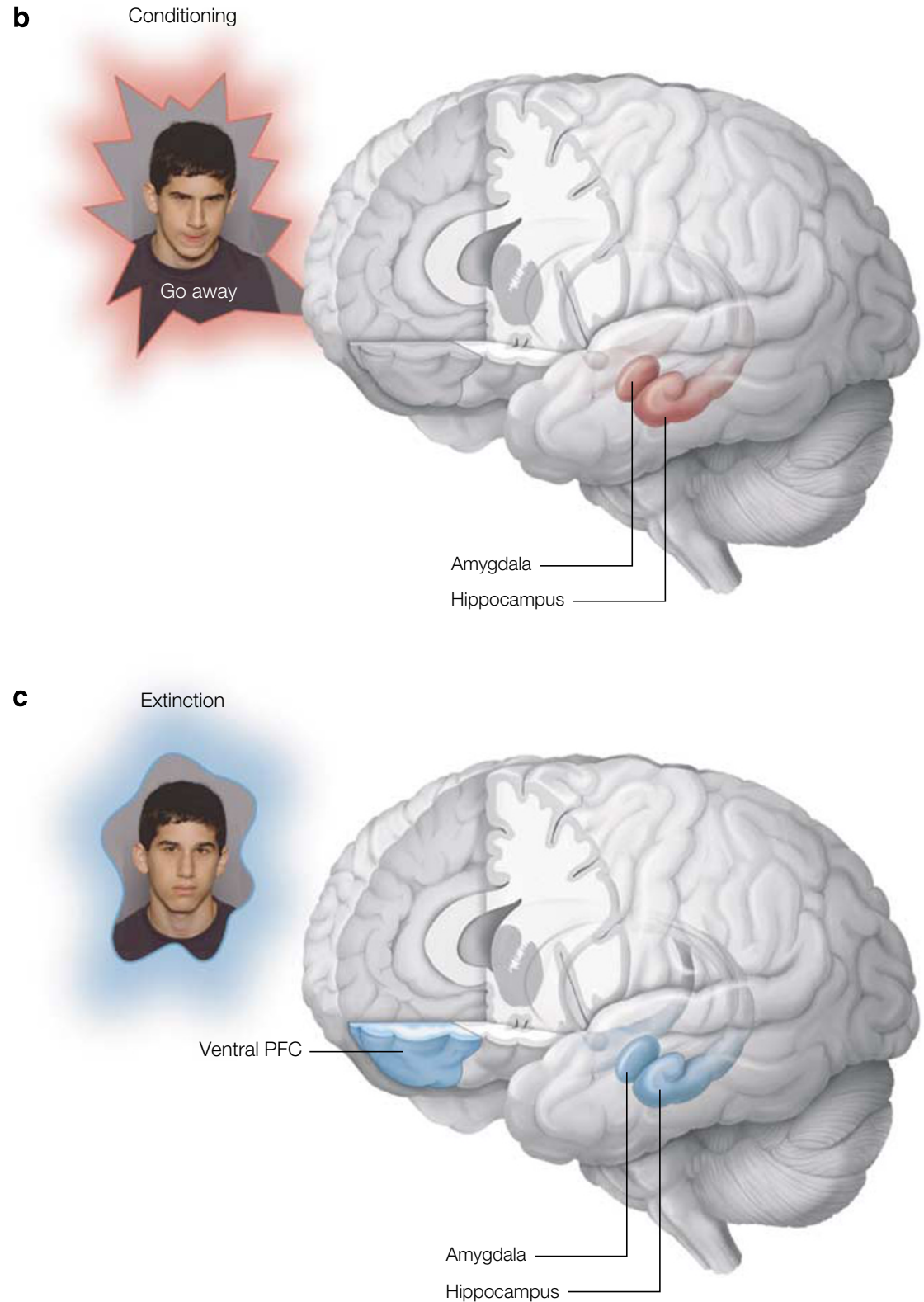

Figure 4. Emerging research implicates conditioning in social phobia. (a) A schematic representation of the experiment in Lissek et al (2008b). This experiment demonstrated perturbed conditioning in social phobia, when neutral conditioned-stimulus (CS) photographs of an individual were paired with unconditioned stimuli (UCS) consisting of angry expressions from the same individual and angry comments. Although the original experiment was conducted using adult photographs and adult patients, Figure 4 relies on adolescent photographs, given relevance for studies in adolescence, a time by which precursors of adult social phobia appear readily apparent. (b) Schematic depiction of neural architecture presumably engaged on presentation of CS paired with the depicted UCS during conditioning. (c) Schematic depiction of neural architecture presumably engaged during CS extinction. 
for novel treatments shown first to alter amygdala-PFC circuitry in rodents and nonhuman primates.

Novel treatments. Perhaps the most promising insights to emerge from translational work on fear-related learning relate to research on therapeutics. Many pharmacological advances capitalize on serendipitous clinical observation. Sophisticated understandings on the neural architecture that supports fear-related learning, in contrast, might generate treatments from neuroscience. Neuroscience provides both relatively broad and more specific insights.

Therapeutic research is shaped broadly by a view of anxiety as reflecting individual differences in learning. From this perspective, anxiety disorders reflect failures in patients to discriminate among cues that vary in terms of their dangerousness (Grillon, 2002; Lissek et al, 2008a). The response to overt danger is appropriately and similarly extreme in patients and healthy subjects, with no betweengroup difference. However, as in the 5HT1ARKO mouse, response to ambiguous danger is abnormal. This view emphasizes the potential therapeutic benefits afforded by teaching anxious children to differentiate scenarios of varying threat intensities.

At a more specific level, a view of individual differences as related to poor discriminative learning generates particular hypotheses. Perhaps the strongest data emerge for extinction, viewed as learning to discriminate among safe, formerly dangerous, and currently dangerous scenarios. Considerable work examines the pharmacology of extinction. Perhaps the best-studied agent is the glutamatergic agonist, D-cycloserine (DCS), which strengthens fear extinction in rodents (Quirk and Mueller, 2008). This effect is thought to emerge by facilitating LTP and other learningrelated processes that allow the organism to precisely differentiate among classes of threat cues based on temporal characteristics of CS-US relationships. Of note, although there are negative data (Guastella et al, 2007; Kushner et al, 2007), at least four studies in adult anxiety disorders demonstrate a clinical effect of DCS on extinction-based learning, as taught to patients using CBT (Rothbaum, 2008). Moreover, the success of this approach generates hypotheses on a host of other novel treatments that might similarly target anxiety by facilitating learning-related processes. These include agents acting on cannabinoid (Pamplona et al, 2006), glutamate (Zushida et al, 2007), and glucocorticoid (Yang et al, 2006) systems.

Finally, data on reconsolidation generate other potential novel insights on therapeutics, as an aversive memory trace may also be uniquely susceptible to disruption during the postretrieval reconsolidation process. Thus, pharmacological (Debiec and LeDoux, 2006) and behavioral (Walker et al, 2003) manipulations suggest fruitful approaches. For example, $\beta$-adrenergic manipulation may block reconsolidation (Debiec and LeDoux, 2006). However, unlike work on DCS in extinction, basic science findings on reconsolidation have not generated convincing data from randomized controlled trials of anxiety disorders.
Importantly, all available research using DCS has been implemented in adult anxiety disorders. Attempts to implement such work in children confront the same complex set of issues delineated above in the discussions of SSRIs and CBT. Thus, in terms of potential for advance, novel treatments might target in young children earlyemerging deficits before chronic anxiety disorders become established. This approach specifically might be encouraged, based on work in rodents and nonhuman primates that relates individual differences in the neural architecture targeted by these treatments to life-long patterns of anxious behavior. On the other hand, ethical considerations might discourage such attempts. Again, as noted above, these considerations apply because children depend on adults to act on their behalf. Moreover, findings in neuroscience demonstrate particularly persistent effects of psychological or pharmacological manipulations that act on neural architecture engaged by fear conditioning. These findings emphasize the need to carefully weigh risks and benefits of manipulating this circuit.

\section{CONCLUSION}

Although considerable data support developmental perspectives, the data on anxiety appear particularly compelling. Longitudinal studies in children establish the relationship between early-emerging and later anxiety. These data parallel findings in rodents and nonhuman primates; together data show that early-life patterns of anxious behavior predict patterns of anxious behavior manifest throughout life. Moreover, emerging translational research implicates a conserved neural architecture in this profile. This architecture encompasses the amygdala, PFC, and hippocampus, to support psychological processes that also exhibit strong cross-species parallels. These consistent themes provide fertile ground for translational work; this includes attempts to extend clinical findings on threat bias through basic work as well as attempts to extend basic findings on fear-related learning through clinical work. Most importantly, however, the consistent themes in this research generate a series of testable hypotheses on novel treatment. Considering approaches for testing these hypotheses precisely elucidates the challenges that confront attempts to capitalize on neuroscience advances afforded by a developmental perspective on therapeutics.

\section{DISCLOSURE/CONFLICT OF INTEREST}

The author(s) declare that, except for income received from the primary employer, no financial support or compensation has been received from any individual or corporate entity over the past 3 years for research or professional service and there are no personal financial holdings that could be perceived as constituting a potential conflict of interest. Dr Pine has received compensation for activities 
related to teaching, editing, and clinical care that pose no conflicts of interest.

\section{ACKNOWLEDGEMENTS}

The ideas in this report strongly reflect the pervasive influences of colleagues and collaborators. Specific viewpoints contained herein were heavily shaped by discussions with Drs Brendan Bradley, Christian Grillon, Shmuel Lissek, Donald F Klein, Rachel G Klein, and Karin Mogg. SMH is supported by an NSF GRFP.

\section{REFERENCES}

Ansorge MS, Morelli E, Gingrich JA (2008). Inhibition of serotonin but not norepinephrine transport during development produces delayed, persistent perturbations of emotional behaviors in mice. J Neurosci 28: 199-207.

Bar-Haim Y, Lamy D, Pergamin L, Bakermans-Kranenburg MJ, van IMH (2007). Threat-related attentional bias in anxious and nonanxious individuals: a metaanalytic study. Psychol Bull 133: 1-24.

Barrett PM, Duffy AL, Dadds MR, Rapee RM (2001). Cognitive-behavioral treatment of anxiety disorders in children: long-term (6-year) follow-up. J Consult Clin Psychol 69: 135-141.

Beck AT (1976). Cognitive Therapy and the Emotional Disorders. International Universities Press: New York

Beesdo K, Bittner A, Pine DS, Stein MB, Hofler M, Lieb R et al (2007). Incidence of social anxiety disorder and the consistent risk for secondary depression in the first three decades of life. Arch Gen Psychiatry 64: 903-912.

Blair J, Mithcell D, Blair K (2005). The Psychopath: Emotion and the Brain. Blackwell: Oxford, United Kingdom.

Bouton ME (2002). Context, ambiguity, and unlearning: sources of relapse after behavioral extinction. Biol Psychiatry 52: 976-986.

Bridge JA, lyengar S, Salary CB, Barbe RP, Birmaher B, Pincus HA et al (2007). Clinical response and risk for reported suicidal ideation and suicide attempts in pediatric antidepressant treatment: a meta-analysis of randomized controlled trials. JAMA 297: 1683-1696.

Caldji C, Diorio J, Anisman H, Meaney MJ (2004). Maternal behavior regulates benzodiazepine/GABAA receptor subunit expression in brain regions associated with fear in BALB/c and C57BL/6 mice. Neuropsychopharmacology 29: 1344-1352.

Carew MB, Rudy JW (1991). Multiple functions of context during conditioning: a developmental analysis. Dev Psychobiol 24: 191-209.

Carola V, Frazzetto G, Pascucci T, Audero E, Puglisi-Allegra S, Cabib S et al (2008). Identifying molecular substrates in a mouse model of the serotonin transporter $\times$ environment risk factor for anxiety and depression. Biol Psychiatry 63: 840-846.

Clarke P, Macleod C, Shirazee N (2008). Prepared for the worst: readiness to acquire threat bias and susceptibility to elevate trait anxiety. Emotion 8: 47-57.

Dandeneau SD, Baldwin MW, Baccus JR, Sakellaropoulo M, Pruessner JC (2007). Cutting stress off at the pass: reducing vigilance and responsiveness to social threat by manipulating attention. J Pers Soc Psychol 93: 651-666.

Davis $M$ (1998). Are different parts of the extended amygdala involved in fear $v s$ anxiety? Biol Psychiatry 44: 1239-1247.

Davis M, Whalen PJ (2001). The amygdala: vigilance and emotion. Mol Psychiatry 6: 13-34.

Debiec J, LeDoux JE (2006). Noradrenergic signaling in the amygdala contributes to the reconsolidation of fear memory: treatment implications for PTSD. Ann NY Acad Sci 1071: 521-524

Dudai Y (2006). Reconsolidation: the advantage of being refocused. Curr Opin Neurobiol 16: 174-178

Duvarci S, Nader K (2004). Characterization of fear memory reconsolidation. J Neurosci 24: 9269-9275.

Eldar S, Ricon T, Bar-Haim Y (2008). Plasticity in attention: implications for stress response in children. Behav Res Ther 46: 450-461.

Fischer A, Sananbenesi F, Schrick C, Spiess J, Radulovic J (2004). Distinct roles of hippocampal de novo protein synthesis and actin rearrangement in extinction of contextual fear. J Neurosci 24: 1962-1966.

Fost $N$ (2001). Ethical issues in research and innovative therapy in children with mood disorders. Biol Psychiatry 49: 1015-1022.

Fox NA, Hane AA, Pine DS (2007). Plasticity for affective neurocircuitry: how the environment affects gene expression. Curr Dir Psychol Sci 16: 1-5.
Frey U, Morris RG (1997). Synaptic tagging and long-term potentiation. Nature 385 533-536.

Grillon C (2002). Associative learning deficits increase symptoms of anxiety in humans. Biol Psychiatry 51: 851-858.

Gross C, Hen R (2004). The developmental origins of anxiety. Nat Rev Neurosci 5 : 545-552.

Gunnar M, Quevedo K (2007). The neurobiology of stress and development. Annu Rev Psychol 58: 145-173.

Guastella AJ, Dadds MR, Lovibond PF, Mitchell P, Richardson R (2007). A randomized controlled trial of the effect of D-cycloserine on exposure therapy for spider fear. J Psychiatr Res 41: 466-471.

Hefner K, Holmes A (2007). Ontogeny of fear-, anxiety- and depression-related behavior across adolescence in C57BL/6J mice. Behav Brain Res 176: 210-215.

Hunt P, Campbell B (1997). Developmental dissociation of the components of conditioned fear. In: Bouton M, Fanselow M (eds). Learning, Motivaiton and Cognition: The Functional Behaviorism of Robert C Bolles. American Psychological Association: Washington, DC pp 53-74.

Hyman SE (2007). Can neuroscience be integrated into the DSM-V? Nat Rev Neurosci 8: 725-732.

Ishiwata H, Shiga T, Okado N (2005). Selective serotonin reuptake inhibitor treatment of early postnatal mice reverses their prenatal stress-induced brain dysfunction. Neuroscience 133: 893-901.

Kagan J (1994). Galen's Prophecy. Basic Books: New York, NY.

Kim JH, Richardson R (2008). The effect of temporary amygdala inactivation on extinction and reextinction of fear in the developing rat: unlearning as a potential mechanism for extinction early in development. J Neurosci 28: 1282-1290.

Kim JJ, Fanselow MS (1992). Modality-specific retrograde amnesia of fear. Science 256: 675-677

Kindt M, Brosschot JF, Everaerd W (1997). Cognitive processing bias of children in a real life stress situation and a neutral situation. J Exp Child Psychol 64 79-97.

Klemenhagen KC, Gordon JA, David DJ, Hen R, Gross CT (2006). Increased fear response to contextual cues in mice lacking the 5-HT1A receptor. Neuropsychopharmacology 31: 101-111.

Kushner MG, Kim SW, Donahue C, Thuras P, Adson D, Kotlyar M et al (2007). D-cycloserine augmented exposure therapy for obsessive-compulsive disorder. Biol Psychiatry 62: 835-838.

Lau JY, Lissek S, Nelson EE, Lee Y, Roberson-Nay R, Poeth K et al (2008). Fear conditioning in adolescents with anxiety disorders: results from a novel experimental paradigm. J Am Acad Child Adolesc Psychiatry 47: 94-102.

LeDoux JE (2000). Emotion circuits in the brain. Annu Rev Neurosci 23: 155-184.

Lissek S, Biggs AL, Rabin S, Cornwell BR, Alvarez RP, Pine DS et al (2008a). Generalization of conditioned fear-potentiated startle in humans: Experimental validation and clinical relevance. Bhav Res Ther 46: 678-687.

Lissek S, Levenson J, Biggs AL, Johnson LL, Ameli R, Pine DS et al (2008b). Elevated fear conditioning to socially relevant unconditioned stimuli in social anxiety disorder. Am J Psychiatry 165: 124-132.

Lissek S, Powers AS, McClure EB, Phelps EA, Woldehawariat G, Grillon C et al (2005). Classical fear conditioning in the anxiety disorders: a meta-analysis. Bhav Res Ther 43: 1391-1424.

MacLeod C, Mathews A, Tata P (1986). Attentional bias in emotional disorders. J Abnorm Psychol 95: 15-20.

MacLeod C, Rutherford E, Campbell L, Ebsworthy G, Holker L (2002). Selective attention and emotional vulnerability: assessing the causal basis of their association through the experimental manipulation of attentional bias. J Abnorm Psychol 111: 107-123.

Malenka RC, Nicoll RA (1999). Long-term potentiation-a decade of progress? Science 285: 1870-1874.

Martin M, Horder P, Jones GV (1992). Integral bias in naming of phobia-related words. Cogn Emotion 6: 479-486.

Maya Vetencourt JF, Sale A, Viegi A, Baroncelli L, De Pasquale R, O FOL et al (2008). The antidepressant fluoxetine restores plasticity in the adult visual cortex. Science 320: 385-388

Meaney MJ (2001). Maternal care, gene expression, and the transmission of individual differences in stress reactivity across generations. Annu Rev Neurosci 24: 1161-1192.

Mogg K, Bradley BP (1998). A cognitive-motivational analysis of anxiety. Behav Res Ther 36: 809-848.

Monk CS, Nelson EE, McClure EB, Mogg K, Bradley BP, Leibenluft E et al (2006). Ventrolateral prefrontal cortex activation and attentional bias in response to angry faces in adolescents with generalized anxiety disorder. Am J Psychiatry 163: 1091-1097.

Monk CS, Telzer EH, Mogg K, Bradley BP, Mai X, Louro HMC et al (2008). Amygdala and ventrolateral prefrontal cortext activation to masked angry faces in 
children and adolescents with generalized anxiety disorder. Arch Gen Psychiatry 65: 568-576.

Nader K, Schafe GE, LeDoux JE (2000). The labile nature of consolidation theory. Nat Rev Neurosci 1: 216-219.

Nelson CA, Bloom FE, Cameron JL, Amaral D, Dahl RE, Pine D (2002). An integrative, multidisciplinary approach to the study of brain-behavior relations in the context of typical and atypical development. Dev Psychopathol 14: 499-520.

Ollendick TH, Yang B, King NJ, Dong Q, Akande A (1996). Fears in American, Australian, Chinese, and Nigerian children and adolescents: a cross-cultural study. J Child Psychol Psychiatry 37: 213-220.

Pamplona FA, Prediger RD, Pandolfo P, Takahashi RN (2006). The cannabinoid receptor agonist WIN 55,212-2 facilitates the extinction of contextual fear memory and spatial memory in rats. Psychopharmacology (Berl) 188: 641-649.

Perez-Edgar K, Fox NA (2005). Temperament and anxiety disorders. Child Adolesc Psychiatr Clin N Am 14: 681-706, viii.

Phelps EA, LeDoux JE (2005). Contributions of the amygdala to emotion processing: from animal models to human behavior. Neuron 48: 175-187.

Phillips RG, LeDoux JE (1992). Differential contribution of amygdala and hippocampus to cued and contextual fear conditioning. Behav Neurosci 106: 274-285.

Pine DS (2002). Treating children and adolescents with selective serotonin reuptake inhibitors: how long is appropriate? J Child Adolesc Psychopharmacol 12 189-203.

Pine DS (2007). Research review: a neuroscience framework for pediatric anxiety disorders. J Child Psychol Psychiatry 48: 631-648.

Quirk GJ, Gehlert DR (2003). Inhibition of the amygdala: key to pathological states? Ann NY Acad Sci 985: 263-272.

Quirk GJ, Mueller D (2008). Neural mechanisms of extinction learning and retrieval. Neuropsychopharmacology 33: 56-72.

Rapee RM, Kennedy S, Ingram M, Edwards S, Sweeney L (2005). Prevention and early intervention of anxiety disorders in inhibited preschool children. $J$ Consult Clin Psychol 73: 488-497.

Rogan MT, Staubli UV, LeDoux JE (1997). Fear conditioning induces associative long-term potentiation in the amygdala. Nature 390: 604-607.

Rothbaum BO (2008). Critical parameters for D-cycloserine enhancement of cognitive-behaviorial therapy for obsessive-compulsive disorder. Am J Psychiatry 165: 293-296.

Rudy JW (1993). Contextual conditioning and auditory cue conditioning dissociate during development. Behav Neurosci 107: 887-891.

Schafe GE, Nader K, Blair HT, LeDoux JE (2001). Memory consolidation of Pavlovian fear conditioning: a cellular and molecular perspective. Trends Neurosci 24: 540-546.
Schmidt RA, Bjork RA (1992). New conceptualizations of practice: common principles in three paradigms suggest new concepts for training. Psychol Sci 3 207-217.

Sevelinges Y, Moriceau S, Holman P, Miner C, Muzny K, Gervais R et al (2007). Enduring effects of infant memories: infant odor-shock conditioning attenuates amygdala activity and adult fear conditioning. Biol Psychiatry 62: 1070-1079.

Shionoya K, Moriceau S, Lunday L, Miner C, Roth TL, Sullivan RM (2006). Development switch in neural circuitry underlying odor-malaise learning. Learn Mem 13: 801-808

Shionoya K, Moriceau S, Bradstock P, Sullivan RM (2007). Maternal attenuation of hypothalamic paraventricular nucleus norepinephrine switches avoidance learning to preference learning in preweanling rat pups. Horm Behav 52 $391-400$.

Sotres-Bayon F, Bush DE, LeDoux JE (2007). Acquisition of fear extinction requires activation of NR2B-containing NMDA receptors in the lateral amygdala. Neuropsychopharmacology 32: 1929-1940.

Sullivan R, Wilson DA, Feldon J, Yee BK, Meyer U, Richter-Levin G et al (2006). The International Society for Developmental Psychobiology annual meeting symposium: impact of early life experiences on brain and behavioral development. Dev Psychobiol 48: 583-602.

Tsetsenis T, Ma XH, Lo lacono L, Beck SG, Gross C (2007). Suppression of conditioning to ambiguous cues by pharmacogenetic inhibition of the dentate gyrus. Nat Neurosci 10: 896-902.

Vianna MR, Igaz LM, Coitinho AS, Medina JH, Izquierdo I (2003). Memory extinction requires gene expression in rat hippocampus. Neurobiol Learn Mem 79 199-203.

Walker MP, Brakefield T, Hobson JA, Stickgold R (2003). Dissociable stages of human memory consolidation and reconsolidation. Nature 425: 616-620.

Waters AM, Mogg K, Bradley BP, Pine DS (2008). Attention bias for emotional faces in children with generalized anxiety disorder. J Am Acad Child Adolesc Psychiatry 47: 435-442.

Williams JM, Mathews A, MacLeod C (1996). The emotional Stroop task and psychopathology. Psychol Bull 120: 3-24.

Wilson EJ, MacLeod C, Mathews A, Rutherford EM (2006). The causal role of interpretive bias in anxiety reactivity. J Abnorm Psychol 115: 103-111.

Yang YL, Chao PK, Lu KT (2006). Systemic and intra-amygdala administration of glucocorticoid agonist and antagonist modulate extinction of conditioned fear Neuropsychopharmacology 31: 912-924.

Zushida K, Sakurai M, Wada K, Sekiguchi M (2007). Facilitation of extinction learning for contextual fear memory by PEPA: a potentiator of AMPA receptors J Neurosci 27: 158-166. 